\title{
Vorticity from irrotationally forced flow
}

\author{
Fabio Del Sordo and Axel Brandenburg \\ NORDITA, Roslagstullsbacken 23, SE-10691 Stockholm, Sweden; and \\ Department of Astronomy, Stockholm University, SE 10691 Stockholm, Sweden
}

\begin{abstract}
In the interstellar medium the turbulence is believed to be forced mostly through supernova explosions. In a first approximation these flows can be written as a gradient of a potential being thus devoid of vorticity. There are several mechanisms that could lead to vorticity generation, like viscosity and baroclinic terms, rotation, shear and magnetic fields, but it is not clear how effective they are, neither is it clear whether the vorticity is essential in determining the turbulent diffusion acting in the ISM. Here we present a study of the role of rotation, shear and baroclinicity in the generation of vorticity in the ISM.
\end{abstract}

Keywords. Galaxies: magnetic fields - ISM: bubbles

The study of the interstellar medium (ISM) is strictly connected with that of supernovae explosions, being this one of the most important phenomena in determining its dynamic. Such events act on scales up to $\sim 100$ pc injecting enough energy to sustain turbulence with velocities of $\sim 10 \mathrm{~km} / \mathrm{s}$. An understanding of the generation of turbulence and vorticity in the ISM is a basic requirement in order to formulate a theory for the production of interstellar magnetic fields. The first step to perform numerical simulations of these explosion is to assume the presence of pure potential forcing. Indeed a supernova explosion can be seen at first glance as a pure spherical expansion that is driving turbulence in the ISM. In our simulations we use the PENCIL CoDE, http://pencil-code.googlecode.com/ We simulate spherical expansions following the work of Mee \& Brandenburg (2006). We analyze flows that are only weakly supersonic and use a constant and uniform viscosity in an unstratified medium. We solve the Navier-Stokes equations in the presence of viscosity and with a potential forcing $\nabla \phi$ where $\phi$ is given by

$$
\phi(\boldsymbol{x}, t)=\phi_{0} N \exp \left\{\left[\boldsymbol{x}-\boldsymbol{x}_{\mathrm{f}}(t)\right]^{2} / R^{2}\right\} .
$$

Here $\boldsymbol{x}=(x, y, z)$ is the position vector, $\boldsymbol{x}_{\mathrm{f}}(t)$ is the random forcing position, $R$ is the radius of the Gaussian, and $N$ is a normalization factor. We consider two forms for the time dependence of $\boldsymbol{x}_{\mathrm{f}}$. First, we take $\boldsymbol{x}_{\mathrm{f}}$ such that the forcing is $\delta$-correlated in time. Second, we include a forcing time $\delta t_{\text {force }}$ that defines the interval during which $\boldsymbol{x}_{\mathrm{f}}$ remains constant. We study then the effect of rotation on such environment. Rotation causes the action of the Coriolis force, $2 \boldsymbol{\Omega} \times \boldsymbol{u}$, in the evolution equation for the velocity. We investigate flows with Reynolds numbers up to 150 . We find that there is a clear production of small scale vorticity when we use both a finite and $\delta$-correlated forcing. In this last case we observe some spurious vorticity also at very low Coriolis number, due probably to some numerical artifact. We then consider the effects of shear on the potential flow. In the presence of linear shear with $\boldsymbol{u}^{S}=(0, S x, 0)$, the evolution equation for the departure from the mean shear attains additional terms, $-\boldsymbol{u}^{S} \boldsymbol{\nabla} \cdot \boldsymbol{u}-\boldsymbol{u} \cdot \boldsymbol{\nabla} \boldsymbol{u}^{S}$. Also in this case we observe the production of vorticity but we do not find clear results for small values of the shear. We have thus observed a production of vorticity due to rotation and shear, but these effects are not able to produce enough vorticity under the physical condition of the ISM, like those described by Beck et al. (1996). When we 

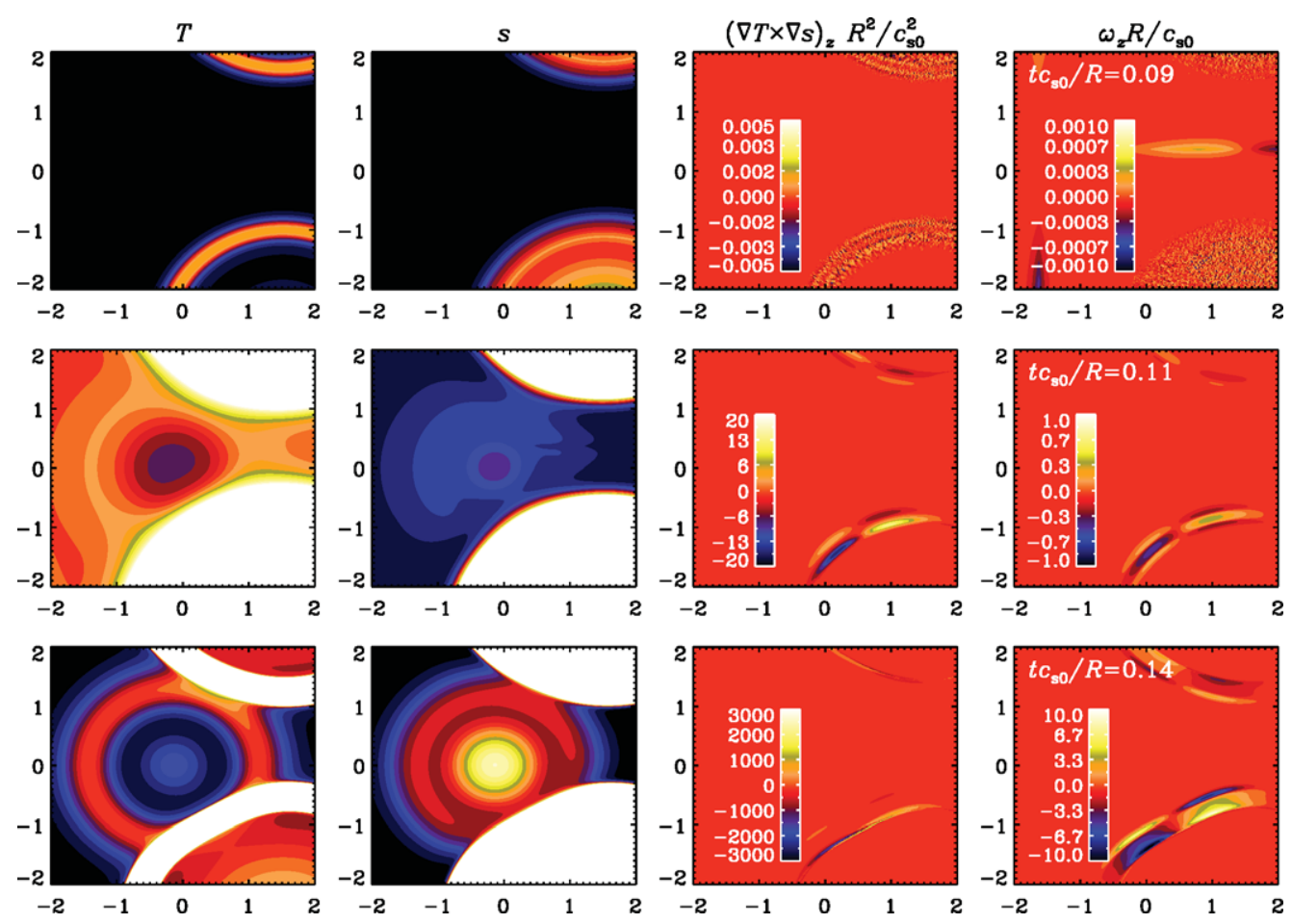

Figure 1. Images of temperature, entropy, baroclinic term $(\nabla T \times \nabla s)_{z}$ and normalized vertical vorticity for a two-dimensional run with $\delta t_{\text {force }} c_{\mathrm{s} 0} / R=0.1$ at an instant shortly before the second expansion wave is launched (top row), and shortly after the second expansion wave is launched (second and third row). In the second and third row the vorticity production from the baroclinic term is clear, while in the top row $(\nabla T \times \nabla s)_{z}$ and $\omega_{z}$ are just at the noise level of the calculation. Shock surfaces are well localized and the zones of maximum production of vorticity are those in which the fronts encounter each other. Adapted from Del Sordo \& Brandenburg (2010).

relax the isothermal condition the system is then also under the action of a baroclinic term. This is proportional to the cross product of the gradients of pressure and density and emerges when taking the curl of the pressure gradient term, $\rho^{-1} \nabla p$. The results we obtain are shown in Figure 1.

It turns then out that the baroclinic term is more efficient in the production of the vorticity. Moreover the biggest amount of vorticity is observed when shock fronts encounter each other.

Speaking about possible dynamo action, as pointed out by Brandenburg \& Del Sordo (2009), the presence of vorticity does not seem to affect the diffusion of magnetic fields differently than a complete irrotational turbulence. Nevertheless the vorticity plays an important role in dynamo processes so it is still important to address the problem of the generation of the vorticity investigating the possible role of other effects.

\section{References}

Beck, R., Brandenburg, A., Moss, D., Shukurov, A., \& Sokoloff, D. 1996, ARA\&A, 34, 155

Brandenburg, A., \& Del Sordo, F. 2009, in Turbulent diffusion and galactic magnetism, ed. Highlights of Astronomy, Vol. 15 (E. de Gouveia Dal Pino), (in press) CUP, arXiv:0910.0072

Del Sordo, F. \& Brandenburg, A. 2010, A\&AA, submitted, arXiv:1008:5281

Mee, A. J. \& Brandenburg, A. 2006, MNRAS, 370, 415 